\title{
Migrated remnant bioresorbable scaffolds in a left main bifurcation lesion: Insights from optical coherence tomography
}

\author{
Jongkwon Seo ${ }^{1,2 *}$, Yongcheol Kim*, Byeong-Keuk Kim², Sung-Jin Hong ${ }^{2}$, \\ Chul-Min $\mathrm{Ahn}^{2}$, Jung-Sun Kim², Deok-Kyu Cho ${ }^{3}$, Young-Guk $\mathrm{Ko}^{2}$, \\ Donghoon $\mathrm{Choi}^{3}$, Myeong-Ki Hong ${ }^{2}$, Yangsoo Jang ${ }^{2}$ \\ ${ }^{1}$ Department of Cardiology, Sanggye-Paik Hospital, \\ Inje University College of Medicine, Seoul, Republic of Korea \\ ${ }^{2}$ Division of Cardiology, Severance Cardiovascular Hospital, \\ Yonsei University College of Medicine, Seoul, Republic of Korea \\ ${ }^{3}$ Division of Cardiology, Department of Internal Medicine, Yonsei University College of Medicine \\ and Cardiovascular Center, Yongin Severance Hospital, Yongin, Republic of Korea
}

A 77-year-old female patient presented with exertional chest pain. Three years before, she had undergone angiography-guided percutaneous coronary intervention $(\mathrm{PCI})$ with $3.5 \times 28 \mathrm{~mm}$ and $3.0 \times 28 \mathrm{~mm}$ everolimus-eluting bioresorbable scaffolds (BRS; Absorb ${ }^{\mathrm{TM}}$; Abbott Vascular, Santa Clara, CA, USA) implanted in the proximal to distal left anterior descending artery (LAD) and a $3.0 \times 18 \mathrm{~mm}$ BRS in the distal left circumflex artery (LCX) (Suppl. Video 1). Angiography demonstrated the patency of the implanted BRSs in the LAD and LCX, but revealed severe stenosis in the proximal LCX and vertical filling defect in the left main (LM) bifurcation lesion (Fig. 1A, Suppl. Video 2). Optical coherence tomography (OCT) demonstrated scaffold remnants in the LM bifurcation lesion that had migrated from the BRS implanted in the proximal LAD (Fig. 1B, C, Suppl. Video 3). Angiography and OCT images might correspond to a case of scaffold dismantling when compared with the previous final angiography. PCI for the LM bifurcation lesion was planned to cover the migrated scaffold remnants because of concerns regarding embolic risk and scaffold thrombosis. The mini-crush technique with two everolimus-eluting stents (EES; Xience Sierra ${ }^{\circledR}$, Abbott Vascular), a $3.5 \times 38 \mathrm{~mm}$ EES implanted from the LM to the LAD and another $3.5 \times 38 \mathrm{~mm}$ EES implanted in the proximal LCX, was performed. After stent optimization with a kissing balloon and proximal optimization techniques, postPCI OCT demonstrated scaffolds well-apposed by the metal struts of the EESs in the LM bifurcation area (Fig. 1D, E, Suppl. Video 4). The final angiography showed good distal flow without residual stenosis (Fig. 1F, Suppl. Video 5).

This case highlights that a superior resolution of OCT enables detection of migrated non-absorbed scaffolds over a 3-year follow-up and demonstrates scaffolds well-apposed by the implanted metal stent.

Conflict of interest: None declared

\footnotetext{
Address for correspondence: Byeong-Keuk Kim, MD, PhD, Division of Cardiology, Severance Cardiovascular Hospital, Yonsei University College of Medicine, 250 Seongsan-ro, Seodaemun-gu, Seoul 03722, Republic of Korea, tel: +82-2-2228-8460, fax: +82-2-2227-7732, e-mail: kimbk@yuhs.ac

Received: 18.02.2020 Accepted: 25.02.2020

*These authors contributed equally to this work.
} 


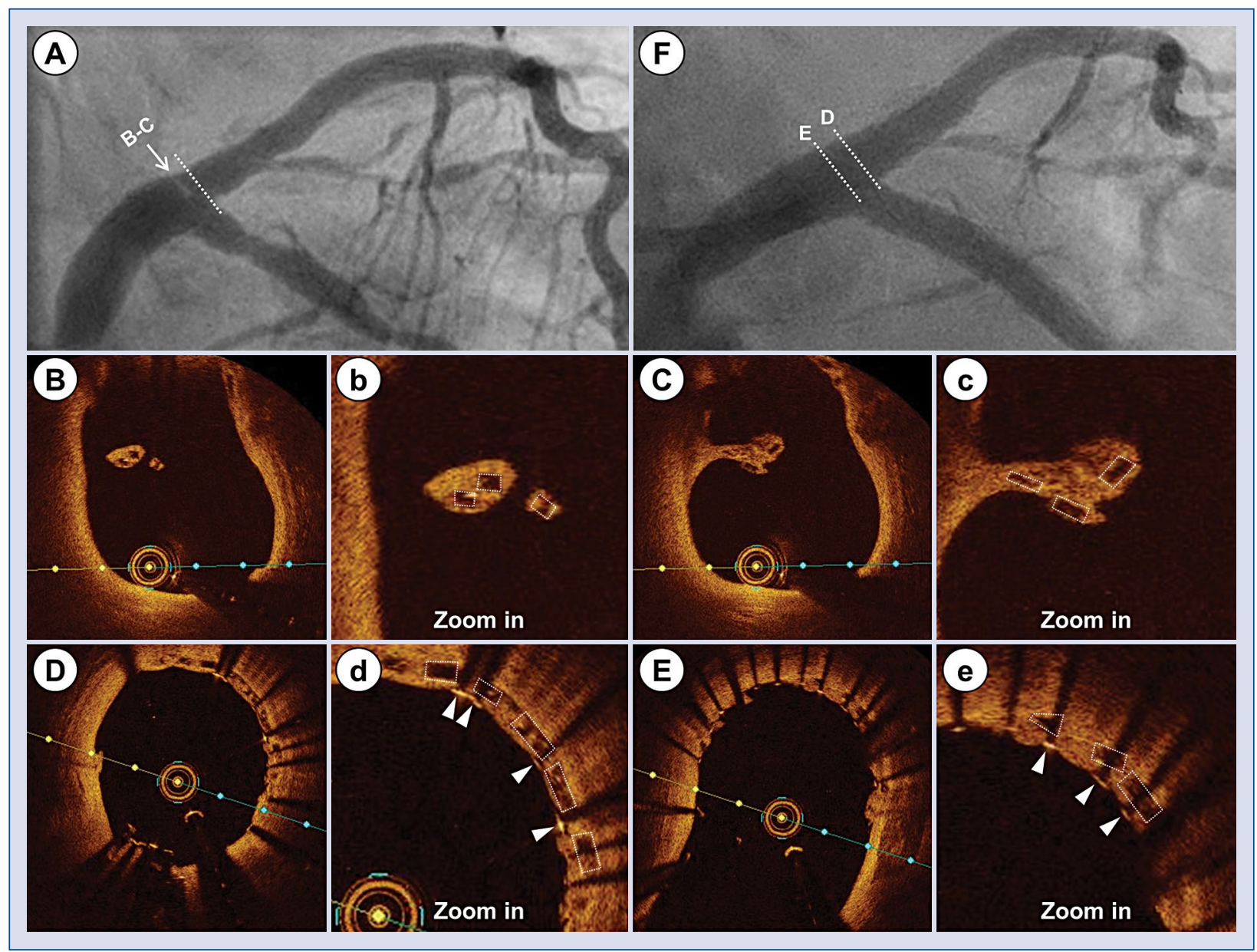

Figure 1. A. Angiography demonstrating vertical filling defect (white arrow) in the left main bifurcation lesion (white dotted line: proximal edge of the previously implanted bioresorbable scaffolds in the left anterior descending artery); B, C. Migrated remnant scaffolds (white boxes in panels b and c) in the left main bifurcation; D, E. Post-percutaneous coronary intervention optical coherence tomography demonstrating scaffolds well-apposed (white boxes in panels $\mathbf{d}$ and $\mathbf{e}$ ) by the struts of the implanted metal stent (arrowheads in panels $\mathbf{d}$ and $\mathbf{e}$ ); F. Final angiography demonstrating successful application of the mini-crush technique with two everolimus-eluting stents. 